\title{
Could sodium imbalances predispose to postoperative venous thromboembolism? An analysis of the NSQIP database
}

\author{
Sally Temraz ${ }^{1}$, Hani Tamim', Aurelie Mailhac ${ }^{2}$ and Ali Taher ${ }^{1 *}$
}

\begin{abstract}
Background: Hyponatremia is common among patients with pulmonary embolism, while hypernatremia increases the risk of venous thromboembolism (VTE). Our objective was to evaluate the association between sodium imbalances and the incidence of VTE and other selected perioperative outcomes.

Methods: We conducted a retrospective cohort study using the American College of Surgeons National Surgical Quality Improvement Program (ACS NSQIP) and identified 1,108,704 patients undergoing major surgery from 2008 to 2012. We evaluated 30-day perioperative outcomes, including mortality and cardiac, respiratory, neurological, urinary, wound, and VTE outcomes. Multivariate logistic regressions were used to estimate the odds of 30-day perioperative outcomes.

Results: Compared with the normal sodium group, in which VTE occurred in 1.0\% of patients, $1.8 \%$ of patients in the hyponatremia group (unadjusted odds ratio (OR) 1.84) and $2.4 \%$ of patients in the hypernatremia group (unadjusted OR 2.49) experienced VTE. Crude mortality was 1.3\% in the normal sodium group, $4.9 \%$ in the hyponatremia group (unadjusted OR 3.93) and $8.4 \%$ in the hypernatremia group (unadjusted OR 7.01). Crude composite morbidity was $7.1 \%$ for the normal sodium group, 16.7\% for the hyponatremia group (unadjusted OR 2.63) and $20.6 \%$ for the hypernatremia group (unadjusted OR 3.43). After adjusting for potential confounders, hyponatremia and hypernatremia remained significantly and independently associated with an increased risk of VTE (adjusted OR 1. 43 and 1.56, respectively), mortality (adjusted OR 1.39 and 1.39, respectively) and composite morbidity (adjusted OR 2. 15 and 3.34 , respectively).
\end{abstract}

Conclusions: Pre-operative hyponatremia and hypernatremia are potential prognostic markers for perioperative 30-day morbidity, mortality and VTE.

Keywords: Hypernatremia, Hyponatremia, Mortality, Morbidity, Surgery, Venous thromboembolism

\section{Background}

Hyponatremia is the most common electrolyte imbalance in hospitalized patients, afflicting at least $30 \%$ of patients in medical, surgical and psychiatric wards $[1,2]$. Hyponatremia is a complicated condition that has been associated with all-cause mortality [3]; length of inpatient stay [4]; gait and attention impairments [5]; bone fractures [6, 7]; and perioperative complications, including 30-day morbidity and mortality $[8,9]$. Most studies

\footnotetext{
* Correspondence: ataher@aub.edu.lb

${ }^{1}$ Department of Internal Medicine, American University of Beirut Medical

Center, Riad El Solh 110 72020, Beirut, Lebanon

Full list of author information is available at the end of the article
}

that have evaluated the outcomes associated with hyponatremia restricted their analyses to hospitalized patients with pre-existing medical conditions, such as congestive heart failure, chronic kidney disease and liver cirrhosis. Although hypernatremia is also associated with increased mortality, most studies have focused on hyponatremia [4]. Hyponatremia is common in patients presenting with acute pulmonary embolism $[10,11]$, while hypernatremia is associated with increased risk of venous thromboembolism (VTE) [12]. To date, no study has evaluated the association between hyponatremia and the occurrence of thromboembolic events. 
VTE includes both deep vein thrombosis and pulmonary embolism and is a major cause of morbidity and mortality worldwide. Approximately 1 in 1000 adults is affected by VTE annually, with the incidence increasing with age [13]. VTE ranks third among cardiovascular diseases, preceded only by coronary artery disease and cerebrovascular disease [14]. The incidence of VTE is increased 100-fold higher in hospitalized patients than in the general population [15], and VTE is detected in approximately $80 \%$ of surgical and medical patients who are not placed on thromboprophylaxis [16].

Our objective was to perform a population-based retrospective cohort study of patients in the American College of Surgeons National Surgical Quality Improvement Program (ACS NSQIP) database to evaluate the association between sodium imbalances and the incidence of VTE and other selected perioperative outcomes.

\section{Methods}

\section{Study design and data collection}

The ACS NSQIP is a nationally validated, outcomebased, risk-adjusted program developed to improve the quality of surgical care for adults in the United States. The program prospectively collects $>150$ variables, including pre- and intraoperative variables and 30-day postoperative mortality and morbidity outcomes for patients undergoing major outpatient or inpatient surgical procedures in more than 200 participating non-Veterans Affairs administration hospitals $[17,18]$. At each hospital site, a trained and certified Surgical Clinical Reviewer (SCR) captures these data using a variety of methods including medical chart abstraction and International Classification of Diseases (ICD) coding. The accuracy of the outcomes of NSQIP dataset are ensured by a host of different training mechanisms for the SCRs and by an Inter-Rater Reliability (IRR) Audit of selected participating sites. Based on the ACS NSQIP participant use files from 2008 to 2012, we identified 1,957,023 adult patients (aged $\geq 18$ years) who underwent major surgery (defined by Current Procedural Terminology codes), No patient was included in the database twice, and only the index case was used for patients who had undergone more than one procedure. We excluded patients who were pregnant and who did not have their sodium levels recorded in their files. Also, due to the high percentages of missing values in a set of confounders, patients with missing values on this set of variables were excluded. A total of 1,108,704 patients were included in the analyses. In accordance with the American University of Beirut's guidelines, which follow the US Code of Federal Regulations for the Protection of Human Subjects, institutional review board approval was not needed or sought for our analysis because the data were collected as part of a quality assurance activity.

\section{Procedures}

Our exposure of interest was the most recent serum sodium measurement within 90 days of surgery. Patients were assigned to one of three groups based on their pre-operative sodium levels. Hyponatremia was defined as sodium $<135 \mathrm{mEq} / \mathrm{L}$, normal sodium levels were between 135 and $145 \mathrm{mEq} / \mathrm{L}$, and hypernatremia was defined as $>145 \mathrm{mEq} / \mathrm{L}$. The primary postoperative outcomes were incidences of VTE (defined as the presence of DVT and/or PE during the postoperative period) and 30-day mortality. DVT is currently defined within ACS NSQIP as the "identification of a new blood clot or thrombus within the venous system", which may be coupled with inflammation within 30 days of the operation. This diagnosis is confirmed by a duplex (ultrasound), venogram or CT scan. The patient must be treated with anticoagulation therapy or placement of a vena cava filter or clipping of the vena cava [19]. PE is defined as "lodging of a blood clot in a pulmonary artery with subsequent obstruction of blood supply to the lung parenchyma". PE is documented if the patient has a V-Q scan interpreted as high probability of PE or a positive CT spiral exam, pulmonary arteriogram or CT angiogram [19]. 30-day mortality is defined as any cause of death (intraoperative or postoperative) occurring within 30 days of surgery. Secondary outcomes included composite comorbidities (wound, cardiac, respiratory, urinary, central nervous system (CNS), sepsis, bleeding, return to operation room and hospital readmission.

\section{Statistical analysis}

Statistical analysis was performed using SAS (SAS version 9.1; SAS, Inc., Cary, NC). P-values were 2sided, and significance was set at 0.05. Baseline demographics and pre-operative and perioperative variables were described for all three categories of blood sodium. Differences were analyzed across categories using the chi-square test for categorical variables and ANOVA for continuous variables. Separate simple and multivariate logistic regression models were used to evaluate the association between blood sodium levels and each outcome. The reference category for the main exposure was set as the normal blood sodium levels. Adjusted odds ratios (ORs) were estimated by including clinically relevant potential confounders of individual outcomes in the models, as outlined in Appendix. Stepwise regression was performed at an entry level of 0.15 and a stay level of 0.25 . To assess effect modification between sodium and VTE, adjusted ORs were estimated by stratifying for age, sex, surgical specialty, steroid use for chronic conditions, body mass index (BMI), presence of active cancer and chemotherapy. 


\section{Results}

For the 1,108,704 patients in this study, the mean age was 57.70 years $(\mathrm{SD}=16.69)$, and $56.2 \%$ of patients were female. Sodium levels were normal in 1,010,167 patients, 87,476 had hyponatremia, and 11,061 had hypernatremia. Table 1 presents demographics and baseline patient characteristics for each of the sodium groups. Compared with patients with normal sodium levels, patients with hyponatremia and hypernatremia were more likely to be older, inpatients, in a high American Society of Anesthesiologists (ASA) class, exposed to prolonged anesthesia, emergency cases, and under a "do not resuscitate" status. Additionally, these patients were more likely to have partially or totally dependent functional status, have lost more than $10 \%$ of their body weight in the prior 6 months, have abnormal pre-operative laboratory studies, and receive perioperative transfusions. Patients with hyponatremia and hypernatremia also had a higher prevalence of dyspnea, diabetes, systemic sepsis, and cardiovascular, respiratory, hepatobiliary, renal, neurological, and hematological-oncological disorders; chronic steroid use; operations within the past month; and infected surgical wounds (Table 1). Patients with normal sodium levels were more likely to be younger than 50 years of age, undergo gynecological, orthopedic, urological and plastic surgeries, have an independent functional status and have a BMI $>35$. Patients with hyponatremia were more likely to be male; have undergone thoracic, vascular and cardiac surgeries; have smoked within 1 year; drink more than two drinks per day; and have a $\mathrm{BMI}<35$. Conversely, patients with hypernatremia were more likely to be non-white, have undergone general and otolaryngology surgeries, have undergone general anesthesia and have been in a coma for more than $24 \mathrm{~h}$ (Table 1).

Thromboembolism occurred in $1.0 \%$ of patients in the normal sodium group compared with $1.8 \%$ in the hyponatremia group (unadjusted OR 1.89, 95\% CI 1.79-2.00) and $2.5 \%$ in the hypernatremia group (unadjusted OR 2.64, 95\% CI 2.34-2.98) (Table 2). Crude mortality was $1.4 \%$ for the normal sodium group compared with $5.1 \%$ for the hyponatremia group (unadjusted OR 3.85, 95\% CI 3.72-3.98) and $9.2 \%$ for the hypernatremia group (unadjusted OR 7.20, 95\% CI 6.73-7.69) (Table 2). Crude composite morbidity was $7.3 \%$ for the normal sodium group compared with $16.9 \%$ for the hyponatremia group (unadjusted OR 2.59, 95\% CI 2.54-2.64) and 22.1\% for the hypernatremia group (unadjusted OR 3.61, 95\% CI 3.45-3.78) (Table 2). Bleeding occurred in $4.3 \%$ of patients with normal sodium levels compared with $9.3 \%$ in those with hyponatremia (unadjusted OR 2.30, 95\% CI 2.24-2.36) and $9.4 \%$ in those with hypernatremia (unadjusted OR 2.32, 95\% CI 2.18-2.48). Of patients with normal sodium levels, $4.5 \%$ had subsequent surgery, and $5.9 \%$ were readmitted; by comparison, among patients with hyponatremia, these values were $9.7 \%$ (unadjusted
OR 2.30, 95\% CI 2.25-2.36) and 9.0\% (unadjusted OR $1.59,95 \%$ CI 1.52-1.67), respectively; and they were 9.1\% (unadjusted OR 2.14, 95\% CI 2.00-2.28) and 6.7\% (unadjusted OR 1.15, 95\% CI 1.00-1.33), respectively, for patients with hypernatremia (Table 2).

After adjusting for potential confounders, hyponatremia was significantly and independently associated with an increased risk of thromboembolism (adjusted OR 1.43, 95\% CI 1.36-1.52), mortality (adjusted OR 1.39, 95\% CI 1.341.45), composite morbidity (adjusted OR 2.15, 95\% CI 2.11-2.19), major bleeding (adjusted OR 1.96, 95\% CI 1.91-2.01), return to operation room (adjusted OR 1.46, 95\% CI 1.42-1.50) and readmission (adjusted OR 1.21, 95\% CI 1.15-1.27) (Table 3). Hypernatremia was also significantly and independently associated with an increased risk of thromboembolism (adjusted OR 1.57, 95\% CI 1.38-1.78), mortality (adjusted OR 1.39, 95\% CI 1.27-1.52), composite morbidity (adjusted OR 3.33, 95\% CI 3.18-3.49), major bleeding (adjusted OR 2.0, 95\% CI 1.87-2.13), and return to operation room (adjusted OR 0.92, 95\% CI 0.80-1.06) (Table 3).

The effect of hyponatremia on thromboembolic outcome was evident across all age groups, both sexes, nonorthopedic patients, patients with or without steroid treatment, patients with a BMI $>18.5$, patients with or without cancer and patients with or without chemotherapy (Table 4). The effect of hypernatremia on thromboembolic outcome was evident across all age groups, both sexes, non-orthopedic patients, patients not on steroids, patients with a BMI $>18.5$, patients with or without cancer and patients not receiving chemotherapy (Table 4).

The median time from blood draw to surgery was 4 days. Because sodium levels vary over time, we performed a restricted sensitivity analysis for patients in whom blood was drawn within 1 week before surgery. Hyponatremia remained significantly and independently associated with an increased risk of thromboembolism, mortality, composite morbidity, major bleeding, return to operation room and readmission (Table 5). Hypernatremia also remained significantly and independently associated with an increased risk of thromboembolism, mortality, composite morbidity, major bleeding and return to operation room.

\section{Discussion}

In this study, we assessed pre-operative hypo- and hypernatremia in patients across all surgical specialties by analyzing data from the ACS NSQIP database. Hyponatremia and hypernatremia were both significantly and independently associated with postoperative thromboembolism, mortality, morbidity, major bleeding and return to operation room. Only hyponatremia was associated with hospital readmission. 
Table 1 Baseline patient characteristics across three categories of blood sodium levels

\begin{tabular}{|c|c|c|c|c|}
\hline & \multicolumn{3}{|c|}{ Sodium (mEq/L) } & \multirow{3}{*}{$p$-value } \\
\hline & $<135$ & $135-145$ & $>145$ & \\
\hline & $(n=87,476)$ & $(n=1,010,167)$ & $(n=11,061)$ & \\
\hline Age & $61.82 \pm 16.52$ & $57.29 \pm 16.65$ & $63.38 \pm 15.89$ & $<0.0001$ \\
\hline$<50$ & $19,723(22.6)$ & $316,094(31.3)$ & $2015(18.2)$ & $<0.0001$ \\
\hline $50-64$ & $26,535(30.3)$ & 330,181 (32.7) & 3500 (31.6) & \\
\hline $65-79$ & $27,788(31.8)$ & $271,429(26.9)$ & $3653(33.0)$ & \\
\hline$\geq 80$ & $13,430(15.4)$ & $92,463(9.2)$ & $1893(17.1)$ & \\
\hline Sex, female & $44,389(50.9)$ & $570,528(56.6)$ & $6142(55.6)$ & $<0.0001$ \\
\hline Race, White & $66,224(84.8)$ & $759,216(84.6)$ & $7777(78.0)$ & $<0.0001$ \\
\hline \multicolumn{5}{|l|}{ Surgery type } \\
\hline General surgery & $54,423(62.2)$ & $656,018(64.9)$ & $7529(68.1)$ & $<0.0001$ \\
\hline Gynecology & $1503(1.7)$ & $35,143(3.5)$ & $242(2.2)$ & \\
\hline Neurosurgery & $2234(2.6)$ & $26,292(2.6)$ & $262(2.4)$ & \\
\hline Orthopedics & $6831(7.8)$ & $87,304(8.6)$ & $782(7.1)$ & \\
\hline Otolaryngology (ENT) & $922(1.1)$ & $15,953(1.6)$ & $206(1.9)$ & \\
\hline Plastics & $615(0.7)$ & $12,522(1.2)$ & $150(1.4)$ & \\
\hline Thoracic & $1289(1.5)$ & $10,221(1.0)$ & $128(1.2)$ & \\
\hline Urology & $2400(2.7)$ & $39,573(3.9)$ & $361(3.3)$ & \\
\hline Vascular & $15,853(18.1)$ & $116,611(11.5)$ & $1317(11.9)$ & \\
\hline Cardiac surgery & 1404 (1.6) & $10,524(1.0)$ & $83(0.8)$ & \\
\hline Principal anesthesia technique, general & $80,842(92.4)$ & $929,863(92.1)$ & $10,238(92.6)$ & $<0.0001$ \\
\hline \multicolumn{5}{|l|}{ ASA classification } \\
\hline$|-| \mid$ & $25,653(29.4)$ & $512,018(50.8)$ & $3642(33.0)$ & $<0.0001$ \\
\hline III & $45,074(51.7)$ & $422,264(41.9)$ & $4666(42.3)$ & \\
\hline IV-V & $16,531(18.9)$ & $73,419(7.3)$ & $2721(24.7)$ & \\
\hline Wound classification, dirty/infected & $17,210(19.7)$ & $59,804(5.9)$ & $1163(10.5)$ & $<0.0001$ \\
\hline Inpatient status & $74,600(85.3)$ & $704,245(69.7)$ & $8451(76.4)$ & $<0.0001$ \\
\hline Emergency case & $25,053(28.6)$ & $123,927(12.3)$ & $2396(21.7)$ & $<0.0001$ \\
\hline Transfusion in $72 \mathrm{~h}$ before surgery & $1992(2.3)$ & $8644(0.9)$ & $643(5.8)$ & $<0.0001$ \\
\hline Do not resuscitate (DNR) status & $1601(1.8)$ & $6425(0.6)$ & $306(2.8)$ & $<0.0001$ \\
\hline \multicolumn{5}{|l|}{ Functional health status before surgery } \\
\hline Independent & $73,530(84.2)$ & $952,051(94.4)$ & $8620(78.1)$ & $<0.0001$ \\
\hline Partially dependent & $10,052(11.5)$ & $42,984(4.3)$ & $978(8.9)$ & \\
\hline Totally dependent & $3708(4.3)$ & $14,080(1.4)$ & $1439(13.0)$ & \\
\hline Dyspnea & $13,284(15.2)$ & $106,592(10.6)$ & $1755(15.9)$ & $<0.0001$ \\
\hline CHF 30 days before surgery & $2530(2.9)$ & $9234(0.9)$ & $443(4.0)$ & $<0.0001$ \\
\hline History of angina 1 month before surgery & $1579(1.8)$ & $10,498(1.0)$ & $163(1.5)$ & $<0.0001$ \\
\hline History of Ml 6 months before surgery & $1650(1.9)$ & $7518(0.7)$ & $267(2.4)$ & $<0.0001$ \\
\hline Previous $\mathrm{PCl}$ & $7353(8.4)$ & $61,587(6.1)$ & $881(8.0)$ & $<0.0001$ \\
\hline Previous cardiac surgery & $8133(9.3)$ & $59,855(5.9)$ & $948(8.6)$ & $<0.0001$ \\
\hline Hypertension requiring medication & $55,678(63.7)$ & $510,100(50.5)$ & $6682(60.4)$ & $<0.0001$ \\
\hline History of revascularization/amputation for peripheral vascular disease & $8298(9.5)$ & $40,414(4.0)$ & $580(5.2)$ & $<0.0001$ \\
\hline Smoking within 1 year & $22,555(25.8)$ & $197,346(19.5)$ & $2045(18.5)$ & $<0.0001$ \\
\hline Current pneumonia & $1537(1.8)$ & $4815(0.5)$ & $514(4.7)$ & $<0.0001$ \\
\hline
\end{tabular}


Table 1 Baseline patient characteristics across three categories of blood sodium levels (Continued)

\begin{tabular}{|c|c|c|c|c|}
\hline & \multicolumn{3}{|c|}{ Sodium (mEq/L) } & \multirow{3}{*}{$p$-value } \\
\hline & $<135$ & $135-145$ & $>145$ & \\
\hline & $(n=87,476)$ & $(n=1,010,167)$ & $(n=11,061)$ & \\
\hline History of severe COPD & $8372(9.6)$ & $52,570(5.2)$ & $967(8.7)$ & $<0.0001$ \\
\hline Ventilator dependent & $1926(2.2)$ & $7756(0.8)$ & $1193(10.8)$ & $<0.0001$ \\
\hline Ascites & $2427(2.8)$ & $6325(0.6)$ & $286(2.6)$ & $<0.0001$ \\
\hline Esophageal varices & $302(0.4)$ & $1017(0.1)$ & $27(0.2)$ & $<0.0001$ \\
\hline Acute renal failure & $1800(2.1)$ & $5066(0.5)$ & $336(3.0)$ & $<0.0001$ \\
\hline Currently on dialysis & $4758(5.4)$ & $18,196(1.8)$ & $353(3.2)$ & $<0.0001$ \\
\hline Impaired sensorium & $2018(2.3)$ & $6524(0.7)$ & $660(6.0)$ & $<0.0001$ \\
\hline Coma $>24 \mathrm{~h}$ & $136(0.2)$ & $596(0.1)$ & $99(0.9)$ & $<0.0001$ \\
\hline History of transient ischemic attacks (TIA) & $3662(4.2)$ & $31,358(3.1)$ & $421(3.8)$ & $<0.0001$ \\
\hline CVA/stroke with neurological deficit & $3432(3.9)$ & $24,497(2.4)$ & $630(5.7)$ & $<0.0001$ \\
\hline CVA/stroke with no neurological deficit & $2941(3.4)$ & $21,936(2.2)$ & $345(3.1)$ & $<0.0001$ \\
\hline Tumor involving CNS & $498(0.6)$ & $3703(0.4)$ & $62(0.6)$ & $<0.0001$ \\
\hline Bleeding disorders & $9989(11.4)$ & $57,672(5.7)$ & $1195(10.8)$ & $<0.0001$ \\
\hline$>10 \%$ loss body weight in previous 6 months & $4158(4.8)$ & $19,804(2.0)$ & $376(3.4)$ & $<0.0001$ \\
\hline Disseminated cancer & $3694(4.2)$ & $21,246(2.1)$ & $329(3.0)$ & $<0.0001$ \\
\hline Chemotherapy $\leq 30$ days pre-operative & $2051(2.3)$ & $14,897(1.5)$ & $239(2.2)$ & $<0.0001$ \\
\hline Radiotherapy in last 90 days & $1011(1.2)$ & $7705(0.8)$ & $100(0.9)$ & $<0.0001$ \\
\hline BMl & $28.40 \pm 8.15$ & $30.31 \pm 8.42$ & $29.57 \pm 8.30$ & $<0.0001$ \\
\hline$<18.5$ & $4085(4.9)$ & $20,342(2.1)$ & $343(3.2)$ & $<0.0001$ \\
\hline $18.5-24.9$ & $27,699(33.1)$ & $249,205(25.3)$ & $2895(27.3)$ & \\
\hline $25.0-29.9$ & $24,643(29.4)$ & $300,443(30.5)$ & $3209(30.3)$ & \\
\hline $30.0-34.9$ & $13,555(16.2)$ & $192,841(19.6)$ & $2068(19.5)$ & \\
\hline$\geq 35.0$ & $13,716(16.4)$ & $221,151(22.5)$ & $2083(19.7)$ & \\
\hline Diabetes mellitus with oral agents or insulin & $23,275(26.6)$ & $167,543(16.6)$ & $2174(19.7)$ & $<0.0001$ \\
\hline Alcohol $>2$ drinks/day 2 wks before admission & $4925(5.6)$ & $25,735(2.6)$ & $323(2.9)$ & $<0.0001$ \\
\hline Open wound/wound infection & $11,046(12.6)$ & $43,031(4.3)$ & $1120(10.1)$ & $<0.0001$ \\
\hline Steroid use for chronic condition & $4717(5.4)$ & $32,860(3.3)$ & $591(5.3)$ & $<0.0001$ \\
\hline Systemic sepsis & $21,684(25.0)$ & $72,666(7.3)$ & $2411(21.9)$ & $<0.0001$ \\
\hline Prior operation within 30 days & $6239(7.1)$ & $25,921(2.6)$ & $960(8.7)$ & $<0.0001$ \\
\hline
\end{tabular}

The reported incidence of hyponatremia upon hospital admission ranges from 5 to $30 \%$ depending on the study population and timing of serum sodium measurements [20-23]. We report a $7.89 \%$ rate of pre-operative hyponatremia among surgical patients. Hyponatremia has been associated with increased mortality in patients with preexisting acute kidney injury [24], chronic kidney disease [25], heart failure [26-29], COPD [30], hip fractures [31], and intracerebral hemorrhage [32]; in patients undergoing cardiac transplantation [33]; and in unselected inpatients with hyponatremia $[9,34,35]$. The reported mortality rate ranges from 5.2 to $22 \%[8,21,22,34,36]$. Our study had a $5.12 \%$ mortality rate among hyponatremic patients, similar to that reported by Leung et al. (5.2\%), Waikar et al. (5.4\%) and Zilberger et al. (5.9\%). However, our mortality rate was lower than that reported by Holland-Bill et al. (8.1\%) and much lower than that reported by Sturdik et al. (22\%). The higher mortality rates reported by Holland-Bill et al. and Sturdik et al. may be related to their study populations, which included patients who were admitted to the internal medicine department. In contrast, our study population and that of Leung et al. involved surgical patients, and studies by Zilberberg et al. and Waiker et al. included a more general patient population. Increased morbidity $[8,28,30]$ and 30-day hospital readmissions $[26,37]$ have also been reported in patients with 
Table 2 Unadjusted analyses for associations between blood sodium levels and outcomes

\begin{tabular}{|c|c|c|c|c|c|c|}
\hline & & & & Unadjusted ORs & & \\
\hline & Sodium (mEq & & & Sodium (mEq/L) & & \\
\hline & $<135$ & $135-145$ & $>145$ & $<135$ & $135-145$ & $>145$ \\
\hline & $(n=87,476)$ & $(n=1,010,167)$ & $(n=11,061)$ & $(n=87,476)$ & $(n=1,010,167)$ & $(n=11,061)$ \\
\hline Thromboembolism & $1577(1.8)$ & $9705(1.0)$ & $276(2.5)$ & $1.89(1.79-2.00)$ & Reference & $2.64(2.34-2.98)$ \\
\hline Mortality & $4479(5.1)$ & $13,972(1.4)$ & $1014(9.2)$ & $3.85(3.72-3.98)$ & Reference & $7.20(6.73-7.69)$ \\
\hline Composite morbidity $^{a}$ & $14,795(16.9)$ & $73,563(7.3)$ & $2442(22.1)$ & $2.59(2.54-2.64)$ & Reference & $3.61(3.45-3.78)$ \\
\hline Wound & $3908(4.5)$ & $23,316(2.3)$ & $439(4.0)$ & $1.98(1.91-2.05)$ & Reference & $1.75(1.59-1.93)$ \\
\hline Cardiac & $1694(1.9)$ & $7472(0.7)$ & $263(2.4)$ & $2.65(2.51-2.80)$ & Reference & $3.27(2.89-3.70)$ \\
\hline Respiratory & $7772(8.9)$ & $31,741(3.1)$ & 1639 (14.8) & $3.01(2.93-3.08)$ & Reference & $5.36(5.08-5.66)$ \\
\hline Urinary & $1724(2.0)$ & $7627(0.8)$ & $378(3.4)$ & $2.64(2.51-2.79)$ & Reference & $4.65(4.19-5.17)$ \\
\hline CNS & $655(0.8)$ & $3938(0.4)$ & $135(1.2)$ & $1.93(1.77-2.10)$ & Reference & $3.16(2.66-3.75)$ \\
\hline Sepsis & $5618(6.4)$ & $25,568(2.5)$ & $870(7.9)$ & $2.64(2.57-2.72)$ & Reference & $3.29(3.06-3.53)$ \\
\hline Bleeding & $8128(9.3)$ & $43,090(4.3)$ & $1036(9.4)$ & $2.30(2.24-2.36)$ & Reference & $2.32(2.18-2.48)$ \\
\hline Return to operation room & $8490(9.7)$ & $45,091(4.5)$ & $1004(9.1)$ & $2.30(2.25-2.36)$ & Reference & $2.14(2.00-2.28)$ \\
\hline Readmission (related) ${ }^{\mathrm{b}}$ & $2062(9.0)$ & $16,099(5.9)$ & $203(6.7)$ & $1.59(1.52-1.67)$ & Reference & $1.15(1.00-1.33)$ \\
\hline
\end{tabular}

${ }^{a}$ Composite morbidity considered positive if any of the following are present: wound, cardiac, respiratory, urinary, CNS injury, sepsis or thromboembolism bample size: 300,815

hyponatremia. Hyponatremia is a common finding among patients with pulmonary embolism, occurring at rates ranging from 21 to $26 \%[10,11]$. In their meta-analysis, Zhou $\mathrm{XY}$ et al. reported that in-hospital mortality was $12.9 \%$ in hyponatremic patients with pulmonary embolism and $2.3 \%$ in normonatremic patients. The mean 30-day mortality was $15.9 \%$ in the hyponatremia group and was $7.4 \%$ in the normonatremia group [38]. However, no study has

Table 3 Adjusted analyses for associations between blood sodium levels and outcomes

\begin{tabular}{|c|c|c|c|}
\hline & \multicolumn{3}{|l|}{ Sodium (mEq/L) } \\
\hline & $<135$ & $135-145$ & $>145$ \\
\hline & $(n=87,476)$ & $(n=1,010,167)$ & $(n=11,061)$ \\
\hline Thromboembolism & $1.43(1.36-1.52)$ & Reference & $1.57(1.38-1.78)$ \\
\hline Mortality & $1.39(1.34-1.45)$ & Reference & $1.39(1.27-1.51)$ \\
\hline Composite morbidity ${ }^{\mathrm{a}}$ & $2.15(2.11-2.19)$ & Reference & $3.33(3.18-3.49)$ \\
\hline Wound & $1.27(1.22-1.32)$ & Reference & $1.25(1.13-1.38)$ \\
\hline Cardiac & $1.24(1.17-1.31)$ & Reference & $1.38(1.21-1.57)$ \\
\hline Respiratory & $1.77(1.72-1.82)$ & Reference & $1.93(1.80-2.07)$ \\
\hline Urinary & $2.28(2.16-2.40)$ & Reference & $3.96(3.56-4.40)$ \\
\hline CNS & $1.45(1.33-1.57)$ & Reference & $2.25(1.89-2.68)$ \\
\hline Sepsis & $1.72(1.67-1.78)$ & Reference & $2.11(1.96-2.28)$ \\
\hline Bleeding & $1.96(1.91-2.01)$ & Reference & $2.00(1.87-2.13)$ \\
\hline $\begin{array}{l}\text { Return to operation } \\
\text { room }\end{array}$ & $1.46(1.42-1.50)$ & Reference & $1.39(1.30-1.49)$ \\
\hline Readmission (related) ${ }^{\mathrm{b}}$ & $1.21(1.15-1.27)$ & Reference & $0.92(0.80-1.06)$ \\
\hline
\end{tabular}

aComposite morbidity considered positive if any of the following are present: wound, cardiac, respiratory, urinary, CNS injury, sepsis or thromboembolism

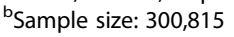

evaluated the direct association between hyponatremia and the development of pulmonary embolism or deep vein thrombosis.

Similar to hyponatremia, hypernatremia was associated with increased mortality in patients undergoing percutaneous endoscopic gastrostomy [39], cardiothoracic surgery [40], or cardiac transplantation; in hip fracture patients [31]; in patients with chronic kidney disease [25]; in critically ill patients [41-43]; and in patients with traumatic brain injuries [33, 44]. The reported mortality rate varies greatly, ranging from as low as $5.2 \%$ to as high as $82 \%$ [45-54]. This discrepancy is mainly due to data from elderly patients $[46,47,49,50]$ and on which cutoff value is used to define hypernatremia [51-54]. However, using a study population similar to ours, Leung et al. reported hypernatremia in $2.2 \%$ of surgical patients compared with $1 \%$ in our population and a mortality rate of $5.2 \%$, which is close to our $9.16 \%$ mortality rate [12]. The authors similarly reported that hypernatremia predicted the occurrence of perioperative major coronary events, pneumonia, and VTE [12]. Our analysis was extended to evaluate the association of both hyponatremia and hypernatremia with the occurrence of thromboembolic events.

Postoperative thromboembolism within a time frame of 30 days occurred in $2.49 \%$ of patients with hypernatremia and $1.8 \%$ of patients with hyponatremia. Leung et al. reported thromboembolism among 1.8\% of hypernatremic patients [12]. We found that the effect of hyponatremia on thromboembolic outcome was evident across all age groups, both sexes, patients 
Table 4 Stratified analyses for associations between blood sodium levels and VTE outcome

\begin{tabular}{|c|c|c|c|}
\hline & \multicolumn{3}{|l|}{ Sodium (mEq/L) } \\
\hline & $<135$ & $135-145$ & $>145$ \\
\hline & $(n=87,476)$ & $(n=1,010,167)$ & $(n=11,061)$ \\
\hline Thromboembolism & $1.43(1.36-1.52)$ & Reference & $1.57(1.38-1.78)$ \\
\hline \multicolumn{4}{|l|}{ Age } \\
\hline$<50(n=337,832)$ & $1.68(1.45-1.94)$ & Reference & $1.65(1.14-2.38)$ \\
\hline $50-64(n=360,216)$ & $1.65(1.49-1.83)$ & Reference & $1.42(1.11-1.82)$ \\
\hline $65-79(n=302,870)$ & $1.28(1.17-1.41)$ & Reference & $1.67(1.37-2.03)$ \\
\hline$\geq 80(n=107,786)$ & $1.17(1.02-1.33)$ & Reference & $1.40(1.06-1.83)$ \\
\hline \multicolumn{4}{|l|}{ Sex } \\
\hline Male $(n=484,893)$ & $1.37(1.27-1.48)$ & Reference & $1.54(1.29-1.84)$ \\
\hline Female $(n=623,811)$ & $1.48(1.36-1.60)$ & Reference & $1.57(1.31-1.88)$ \\
\hline \multicolumn{4}{|l|}{ Surgical specialty } \\
\hline Non-orthopedic $(n=1,013,787)$ & $1.47(1.39-1.56)$ & Reference & $1.62(1.43-1.85)$ \\
\hline Orthopedic $(n=94,917)$ & $1.10(0.89-1.37)$ & Reference & $0.71(0.34-1.51)$ \\
\hline \multicolumn{4}{|l|}{ Steroid use for chronic condition } \\
\hline No $(n=1,070,536)$ & $1.45(1.37-1.54)$ & Reference & $1.66(1.46-1.89)$ \\
\hline Yes $(n=38,168)$ & $1.26(1.06-1.51)$ & Reference & $0.86(0.53-1.39)$ \\
\hline \multicolumn{4}{|l|}{ BMl } \\
\hline$<18.5(n=24,770)$ & $1.28(1.00-1.63)$ & Reference & $1.68(0.92-3.07)$ \\
\hline $18.5-24.9(n=279,799)$ & $1.51(1.36-1.66)$ & Reference & $1.52(1.19-1.95)$ \\
\hline $25.0-29.9(n=328,295)$ & $1.44(1.30-1.60)$ & Reference & $1.50(1.17-1.93)$ \\
\hline $30.0-34.9(n=238,890)$ & $1.30(1.15-1.48)$ & Reference & $1.45(1.12-1.89)$ \\
\hline$\geq 35.0(n=236,950)$ & $1.52(1.32-1.74)$ & Reference & $1.82(1.39-2.38)$ \\
\hline \multicolumn{4}{|l|}{ Presence of active cancer ${ }^{a}$} \\
\hline No $(n=1,068,670)$ & $1.42(1.34-1.51)$ & Reference & $1.53(1.34-1.74)$ \\
\hline Yes $(n=40,034)$ & $1.25(1.04-1.49)$ & Reference & $1.55(1.29-1.85)$ \\
\hline \multicolumn{4}{|c|}{ Chemotherapy for malignancy $\leq 30$ days pre-surgery } \\
\hline No $(n=1,091,517)$ & $1.42(1.34-1.51)$ & Reference & $1.57(1.38-1.78)$ \\
\hline Yes $(n=17,187)$ & $1.67(1.29-2.16)$ & Reference & $1.57(0.79-3.13)$ \\
\hline
\end{tabular}

with or without steroid treatment, patients with a BMI > 18.5, patients with or without cancer and patients with or without chemotherapy. However, hyponatremia was not associated with thromboembolic events in orthopedic patients. The effect of hypernatremia on thromboembolic outcome was also evident across all age groups, both sexes, patients with a BMI > 18.5 , and patients with or without cancer. However, hypernatremic patients undergoing orthopedic surgery and patients on chemotherapy and steroids did not develop thromboembolisms. VTE is a serious complication following major orthopedic surgery, and all patients undergoing orthopedic surgery receive thromboprophylaxis with a pharmacological agent or Intermittent Pneumatic Compression Device (IPCD) for a minimum of 10 to 14 days, sometimes extending prophylaxis up to 35 days [55]. This prophylaxis could explain why hyponatremic and hypernatremic patients undergoing orthopedic surgery were not at risk of developing VTE. Systemic glucocorticoid use increases the risk of VTE among present, new, continuing and recent users but not among former users [56]. Corticosteroid therapy is associated with a nearly 5fold increase in the risk of VTE [57]. Surgical patients with prolonged pre-operative glucocorticoid intake are at a higher risk of developing postoperative VTE and secondary outcomes, including all-cause mortality, urinary tract complications sepsis, wound occurrences, cardiac and respiratory adverse events [58]. This observation suggests that hyponatremic and hypernatremic patients on steroids are at risk for VTE. 
Table 5 Sensitivity analysis of outcomes for Na levels taken $\leq 7$ days prior to surgery

\begin{tabular}{|c|c|c|c|}
\hline & \multicolumn{3}{|l|}{ Sodium (mEq/L) } \\
\hline & $<135$ & $135-145$ & $>145$ \\
\hline & $(n=71,783)$ & $(n=646,416)$ & $(n=7795)$ \\
\hline Thromboembolism & $1.40(1.32-1.49)$ & Reference & $1.66(1.46-1.89)$ \\
\hline Mortality & $1.36(1.30-1.41)$ & Reference & $1.40(1.28-1.53)$ \\
\hline Composite morbidity $^{a}$ & $2.04(2.00-2.08)$ & Reference & $3.86(3.67-4.06)$ \\
\hline Wound & $1.27(1.22-1.32)$ & Reference & $1.28(1.15-1.43)$ \\
\hline Cardiac & $1.24(1.17-1.31)$ & Reference & $1.42(1.24-1.62)$ \\
\hline Respiratory & $1.67(1.62-1.72)$ & Reference & $1.96(1.82-2.11)$ \\
\hline Urinary & $2.07(1.96-2.19)$ & Reference & $4.22(3.78-4.70)$ \\
\hline CNS & $1.36(1.24-1.49)$ & Reference & $2.34(1.95-2.81)$ \\
\hline Sepsis & $1.65(1.59-1.70)$ & Reference & $2.20(2.04-2.38)$ \\
\hline Bleeding & $1.81(1.76-1.86)$ & Reference & $2.10(1.96-2.26)$ \\
\hline $\begin{array}{l}\text { Return to operation } \\
\text { room }\end{array}$ & $1.43(1.39-1.47)$ & Reference & $1.47(1.37-1.58)$ \\
\hline Readmission (related) ${ }^{\mathrm{b}}$ & $1.19(1.12-1.25)$ & Reference & $0.87(0.73-1.03)$ \\
\hline
\end{tabular}

${ }^{a}$ Composite morbidity considered positive if any of the following are present: wound, cardiac, respiratory, urinary, CNS injury, sepsis or thromboembolism ${ }^{\mathrm{b}}$ Sample size: 189,828

However, hypernatremic patients on steroids did not show an increased risk of VTE in our study. Hypernatremia is sometimes encountered in patients with hypertension secondary to aldosteronism [59]. Medical treatment involves the use spironolactone (aldosterone antagonist or Amiloride) in addition to angiotensin-converting enzyme inhibitors for better control of blood pressure [60]. Chae et al. recently reported that after controlling for factors related to VTE, the use of renin-angiotensin inhibitors was still associated with a significantly lower risk of developing VTE [61], which may explain why hypernatremic patients in our study receiving steroids were protected against VTE.

Chemotherapy use in cancer patients increases the risk of VTE 6.5-fold compared with non-cancer patients [62]. Salahudeen et al. recently reported that $90 \%$ of hypernatremia cases among cancer patients are hospital acquired and largely involve leukemia and stem cell transplant patients. The authors also found that, compared with patients with normo- or hyponatremia, patients with hypernatremia were extremely sick and frequently admitted to critical care units [63]. Our results reveal that hypernatremic patients receiving chemotherapy were not at risk of developing VTE, which may be attributed to this high-risk population receiving thromboprophylaxis treatment. The guidelines on VTE prevention in oncology from the United States National Comprehensive Cancer Network $[64,65]$ and the American Society of Clinical Oncology [66] suggest that thromboprophylaxis should be considered for high-risk ambulatory patients with cancer who receive chemotherapy.

The limitations of this work include a current shortage of biological data on the plausibility of the link between hypernatremia and postoperative thromboembolism. The association between hyponatremia and pulmonary embolism has been established. However, this association has not been established for deep vein thrombosis. Second, in the ACS NSQIP database, only one preoperative serum sodium value is available and there was large variability in the collection and timing of preoperative blood work. However, sensitivity analyses suggested that risks were similar in patients with sodium measurements taken within 1 week of surgery. Third, patients are followed after surgery for 30 days thus omplications or death after that period are not included. Fourth, around 260,123 (19.0\%) individuals out of $1,368,827$ were excluded due to missing values on sodium levels. This selection bias has not been accounted for in this study.. Fifth, patients with severe cases of both hyponatremia and hypernatremia likely received some form of treatment. Thus, the incidence of hyponatremia and hypernatremia reported in our study may not be representative of the rates on the day of surgery. Finally, since this study aims at studying causality between sodium imbalances and VTE using a large retrospective dataset, much confounding remains unaddressed despite our careful adjustment for many clinically and statistically relevant factors. For instance, we cannot exclude the possibility of unmeasured confounding factors such as the use of diuretics and DVT prophylaxis, which is unavailable in the ACS NSQIP database analyzed. Thus, it is still not clear whether the associations between both hyponatremia and hypernatremia and increased risk of VTE, morbidity and mortality are due to the adverse effects of sodium imbalances or the underlying diseases. Future work needs to be performed to establish whether sodium imbalance is in fact a causal factor for postoperative VTE.

\section{Conclusion}

Our results reveal an increased VTE risk, as well as increased mortality and morbidity, in both hyponatremic and hypernatremic patients. Because sodium levels are routinely measured in hospitalized patients, they could be easily utilized in identifying patients at risk of developing VTE. Recent evidence suggests that an improvement in serum sodium in hyponatremic patients is associated with a reduction of overall mortality [67]. Thus, hypernatremia and hyponatremia in surgical patients should not be ignored and patients with sodium imbalances ought to be more closely monitored for potential complications after surgery. 


\section{Appendix}

Table 6 Clinically relevant potential confounders

\begin{tabular}{|c|c|c|c|c|c|c|c|c|c|c|c|c|}
\hline & VTE & Mortality & Wound & Cardiac & Respiratory & Urinary & CNS & Sepsis & Bleeding & $\begin{array}{l}\text { Composite } \\
\text { Morbidity }\end{array}$ & $\begin{array}{l}\text { Return } \\
\text { OR }\end{array}$ & Readmission \\
\hline Age & $x$ & $x$ & & $x$ & & $x$ & $x$ & $x$ & & & & $x$ \\
\hline Gender & & & & $x$ & & & & & & & & \\
\hline Race & $x$ & & & & & & & & & & & \\
\hline Type of Anesthesia & $X$ & $x$ & & $x$ & & & & & & & & \\
\hline ASA Class & & $x$ & & $x$ & $x$ & & & & & & $x$ & $x$ \\
\hline Infected Surgical Wound Class & & $x$ & $x$ & & & & & & & & $x$ & $x$ \\
\hline Mean Total Operation Time min & $x$ & & & & & & & & & & & \\
\hline Intraop Transfusions & $x$ & $x$ & $x$ & $x$ & $x$ & & & $x$ & & & & \\
\hline CHF w/in 30 days & $x$ & $x$ & & $x$ & $x$ & & & & & & & $x$ \\
\hline MI w/in 6 months & $x$ & $x$ & & $x$ & & & & & & & & \\
\hline History of angina & & $x$ & & $x$ & & & & & & & & \\
\hline Tobacco use in past year & $x$ & $x$ & & $x$ & $x$ & & $x$ & & & $x$ & & \\
\hline History of severe COPD & & $x$ & & & $x$ & & & & & $x$ & & $x$ \\
\hline $\begin{array}{l}\text { Esophageal varices in previous } \\
6 \text { months }\end{array}$ & & $x$ & & & & & & & $x$ & $x$ & & \\
\hline Acute renal failure & $x$ & $x$ & & & & & & & & & & \\
\hline Currently on dialysis & & $x$ & & $x$ & $x$ & & & $x$ & $x$ & $x$ & & $x$ \\
\hline $\begin{array}{l}\text { Impaired sensorium in } \\
\text { previous } 48 \mathrm{~h}\end{array}$ & & $x$ & & & $x$ & & & & & & & \\
\hline Hemiplegia & $x$ & $x$ & & & & & & & & & & \\
\hline $\begin{array}{l}\text { History of transient ischemic } \\
\text { attacks }\end{array}$ & & $x$ & & & & & $x$ & & & & & \\
\hline $\begin{array}{l}\text { History of CVA with neurological } \\
\text { deficit }\end{array}$ & $x$ & $x$ & & & & & $x$ & & & $x$ & & \\
\hline $\begin{array}{l}\text { History of CVA without } \\
\text { neurological deficit }\end{array}$ & & $x$ & & & & & & & & & & \\
\hline Tumor involving CNS & $x$ & $x$ & & & & & $x$ & & & & & \\
\hline Bleeding disorder & & $x$ & & & & & & & $x$ & & $x$ & $x$ \\
\hline $\begin{array}{l}\text { Weight loss }>10 \% \text { in previous } \\
6 \text { months }\end{array}$ & & $x$ & & & & & & & & & & \\
\hline Disseminated cancer & $x$ & $x$ & & $x$ & $x$ & & & $x$ & & $x$ & & $x$ \\
\hline $\begin{array}{l}\text { Chemotherapy in previous } \\
30 \text { days }\end{array}$ & $x$ & & & & & & & & & & & \\
\hline BMI & $x$ & $x$ & & $x$ & $x$ & $x$ & $x$ & & & $x$ & & $x$ \\
\hline Diabetic on oral drugs or insulin & & $x$ & $x$ & $x$ & & $x$ & $x$ & $x$ & & $x$ & & \\
\hline Hypertension requiring meds & & $x$ & & $x$ & & & $x$ & & & & & \\
\hline Peripheral vascular disease & & $x$ & $x$ & & & & & & & & & \\
\hline Rest pain or gangrene & $x$ & $x$ & & & & & & $x$ & & & & \\
\hline $\begin{array}{l}\text { Open wound (with or without } \\
\text { infection) }\end{array}$ & & & $x$ & & & & & $x$ & & & $x$ & $x$ \\
\hline $\begin{array}{l}\text { Steroid use for chronic } \\
\text { condition }\end{array}$ & $x$ & & & & & & & $x$ & & & & \\
\hline Systemic sepsis in previous $48 \mathrm{~h}$ & & $x$ & & & & & & & & & & \\
\hline WBC count & & & & & & & & X & & & & \\
\hline
\end{tabular}


Table 6 Clinically relevant potential confounders (Continued)

\begin{tabular}{llllll}
\hline & VTE & Mortality Wound Cardiac Respiratory Urinary CNS Sepsis Bleeding Composite Return Readmission \\
OR
\end{tabular}

\section{Abbreviations}

ACS NSQIP: American College of Surgeons National Surgical Quality Improvement Program; ASA: American Society of Anesthesiologists; BMI: Body mass index; CNS: Central nervous system; ICD: International Classification of Diseases; IPCD: Intermittent pneumatic compression device; SCR: Surgical Clinical Reviewer; VTE: Venous thromboembolism

\section{Acknowledgements}

Not applicable.

\section{Funding}

The work under consideration did not receive any funding.

\section{Availability of data and materials}

The data that support the findings of this study are available from [ACS NSQIP: American College of Surgeons National Surgical Quality Improvement Program] but restrictions apply to the availability of these data, which were used under license for the current study, and so are not publicly available. Data are however available from the authors upon reasonable request and with permission of [ACS NSQIP].

\section{Authors' contributions}

ST conceived of the study, wrote the paper and analyzed the data. HT and AM obtained the data and performed the statistical analysis. AT conceived of the study and reviewed and edited the manuscript. All authors read and approved the final manuscript.

\section{Ethics approval and consent to participate}

In accordance with the American University of Beirut's guidelines, which follow the US Code of Federal Regulations for the Protection of Human Subjects, institutional review board approval was not needed or sought for our analysis because the data were collected as part of a quality assurance activity.

\section{Consent for publication}

Not applicable.

\section{Competing interests}

ST reports having received lecture fees from Mercke and travel support from MSD and Roche. AT reports having received honorarium from Novartis Pharmaceuticals and research funding from Novartis Pharmaceuticals and Celgene Corporations. For the remaining authors, none were declared.

\section{Publisher's Note}

Springer Nature remains neutral with regard to jurisdictional claims in published maps and institutional affiliations.

\section{Author details}

${ }^{1}$ Department of Internal Medicine, American University of Beirut Medical Center, Riad El Solh 110 72020, Beirut, Lebanon. ${ }^{2}$ Clinical Research Institute, American University of Beirut Medical Center, Beirut, Lebanon.

Received: 10 August 2017 Accepted: 1 March 2018

Published online: 03 July 2018

\section{References}

1. Hawkins RC. Age and gender as risk factors for hyponatremia and hypernatremia. Clin Chim Acta. 2003;337:169-72

2. Upadhyay A, Jaber BL, Madias NE. Incidence and prevalence of hyponatremia. Am J Med. 2006;119:S30-5.

3. Liamis G, Rodenburg EM, Hofman A, Zietse R, Stricker BH, Hoorn EJ. Electrolyte disorders in community subjects: prevalence and risk factors. Am J Med. 2013;126:256-63.

4. Wald R, Jaber BL, Price LL, Upadhyay A, Madias NE. Impact of hospitalassociated hyponatremia on selected outcomes. Arch Intern Med. 2010;170: 294-302.

5. Renneboog B, Musch W, Vandemergel X, Manto MU, Decaux G. Mild chronic hyponatremia is associated with falls, unsteadiness, and attention deficits. Am J Med. 2006;119:71.e1-8.

6. Kinsella S, Moran S, Sullivan MO, Molloy MG, Eustace JA. Hyponatremia independent of osteoporosis is associated with fracture occurrence. Clin J Am Soc Nephrol. 2010;5:275-80.

7. Ayus JC, Moritz ML. Bone disease as a new complication of hyponatremia: moving beyond brain injury. Clin J Am Soc Nephrol. 2010;5:167-8. 
8. Leung AA, McAlister FA, Rogers SO Jr, Pazo V, Wright A, Bates DW Preoperative hyponatremia and perioperative complications. Arch Intern Med. 2012;172:1474-81.

9. Mohan S, Gu S, Parikh A, Radhakrishnan J. Prevalence of hyponatremia and association with mortality: results from NHANES. Am J Med. 2013;126:1127-37.e1.

10. Scherz N, Labarere J, Mean M, Ibrahim SA, Fine MJ, Aujesky D. Prognostic importance of hyponatremia in patients with acute pulmonary embolism. Am J Respir Crit Care Med. 2010;182:1178-83.

11. Tamizifar B, Kheiry S, Fereidoony F. Hyponatremia and 30 days mortality of patients with acute pulmonary embolism. J Res Med Sci. 2015;20:777-81.

12. Leung AA, McAlister FA, Finlayson SR, Bates DW. Preoperative hypernatremia predicts increased perioperative morbidity and mortality. Am J Med. 2013;126:877-86.

13. White $\mathrm{RH}$. The epidemiology of venous thromboembolism. Circulation. 2003;107:14-8

14. Silverstein MD, Heit JA, Mohr DN, Petterson TM, O'Fallon WM, Melton LJ 3rd. Trends in the incidence of deep vein thrombosis and pulmonary embolism: a 25-year population-based study. Arch Intern Med. 1998;158:585-93.

15. Heit JA, Melton $\sqcup J$ 3rd, Lohse CM, Petterson TM, Silverstein MD, Mohr DN, O'Fallon WM. Incidence of venous thromboembolism in hospitalized patients vs community residents. Mayo Clin Proc. 2001;76:1102-10.

16. Guyatt GH, Eikelboom JW, Gould MK, Garcia DA, Crowther M, Murad MH, Kahn SR, Falck-Ytter Y, Francis CW, Lansberg MG, et al. Approach to outcome measurement in the prevention of thrombosis in surgical and medical patients: antithrombotic therapy and prevention of thrombosis, 9th ed: American College of Chest Physicians Evidence-Based Clinical Practice Guidelines. Chest. 2012;141:e185S-94S.

17. Khuri SF, Henderson WG, Daley J, Jonasson O, Jones RS, Campbell DA Jr, Fink AS, Mentzer RM Jr, Steeger JE. Principal site investigators of the patient safety in surgery S. The patient safety in surgery study: background, study design, and patient populations. J Am Coll Surg. 2007;204:1089-102.

18. Fink AS, Campbell DA Jr, Mentzer RM Jr, Henderson WG, Daley J, Bannister J, Hur K, Khuri SF. The National Surgical Quality Improvement Program in non-veterans administration hospitals: initial demonstration of feasibility. Ann Surg. 2002;236:344-53. discussion 53-4

19. De Martino RR, Goodney PP, Spangler EL, Wallaert JB, Corriere MA, Rzucidlo EM, Walsh DB, Stone DH. Variation in thromboembolic complications among patients undergoing commonly performed cancer operations. J Vasc Surg. 2012;55:1035-40.e4.

20. Zilberberg MD, Exuzides A, Spalding J, Foreman A, Jones AG, Colby C, Shorr AF. Epidemiology, clinical and economic outcomes of admission hyponatremia among hospitalized patients. Curr Med Res Opin. 2008;24 1601-8.

21. Sturdik I, Adamcova M, Kollerova J, Koller T, Zelinkova Z, Payer J. Hyponatraemia is an independent predictor of in-hospital mortality. Eur J Intern Med. 2014;25:379-82.

22. Waikar SS, Mount DB, Curhan GC. Mortality after hospitalization with mild, moderate, and severe hyponatremia. Am J Med. 2009;122:857-65.

23. Frenkel WN, van den Born BJ, van Munster BC, Korevaar JC, Levi M, de Rooij SE. The association between serum sodium levels at time of admission and mortality and morbidity in acutely admitted elderly patients: a prospective cohort study. J Am Geriatr Soc. 2010;58:2227-8.

24. Lee SW, Baek SH, Ahn SY, Na KY, Chae DW, Chin HJ, Kim S. The effects of pre-existing hyponatremia and subsequent-developing acute kidney injury on in-hospital mortality: a retrospective cohort study. PLoS One. 2016;11: e0162990.

25. Kovesdy CP, Lott EH, Lu JL, Malakauskas SM, Ma JZ, Molnar MZ, KalantarZadeh K. Hyponatremia, hypernatremia, and mortality in patients with chronic kidney disease with and without congestive heart failure. Circulation. 2012;125:677-84

26. De Vecchis R, Di Maio M, Di Biase G, Ariano C. Effects of hyponatremia normalization on the short-term mortality and Rehospitalizations in patients with recent acute decompensated heart failure: a retrospective study. J Clin Med. 2016:5:92.

27. Saepudin S, Ball PA, Morrissey H. Hyponatremia during hospitalization and in-hospital mortality in patients hospitalized from heart failure. BMC Cardiovasc Disord. 2015;15:88.

28. Bhavnani SP, Kumar A, Coleman Cl, Guertin D, Yarlagadda RK, Clyne CA Kluger J. The prognostic impact of pre-implantation hyponatremia on morbidity and mortality among patients with left ventricular dysfunction and implantable cardioverter-defibrillators. Europace. 2014;16:47-54.
29. Sato N, Gheorghiade M, Kajimoto K, Munakata R, Minami Y, Mizuno M, Aokage T, Asai K, Sakata Y, Yumino D, et al. Hyponatremia and in-hospital mortality in patients admitted for heart failure (from the ATTEND registry). Am J Cardiol. 2013:111:1019-25.

30. Chalela R, Gonzalez-Garcia JG, Chillaron JJ, Valera-Hernandez L, MontoyaRangel C, Badenes D, Mojal S, Gea J. Impact of hyponatremia on mortality and morbidity in patients with COPD exacerbations. Respir Med. 2016;117:237-42.

31. Madsen CM, Jantzen C, Lauritzen JB, Abrahamsen B, Jorgensen HL. Hyponatremia and hypernatremia are associated with increased 30-day mortality in hip fracture patients. Osteoporos Int. 2016;27:397-404.

32. Kuramatsu JB, Bobinger T, Volbers B, Staykov D, Lucking H, Kloska SP, Kohrmann M, Huttner HB. Hyponatremia is an independent predictor of inhospital mortality in spontaneous intracerebral hemorrhage. Stroke. 2014;45: 1285-91.

33. Maggiore U, Picetti E, Antonucci E, Parenti E, Regolisti G, Mergoni M, Vezzani A, Cabassi A, Fiaccadori E. The relation between the incidence of hypernatremia and mortality in patients with severe traumatic brain injury. Crit Care. 2009;13:R110.

34. Holland-Bill L, Christiansen CF, Heide-Jorgensen U, Ulrichsen SP, Ring T, Jorgensen JO, Sorensen HT. Hyponatremia and mortality risk: a Danish cohort study of 279508 acutely hospitalized patients. Eur J Endocrinol. 2015;173:71-81.

35. Selmer C, Madsen JC, Torp-Pedersen C, Gislason GH, Faber J. Hyponatremia, all-cause mortality, and risk of cancer diagnoses in the primary care setting: a large population study. Eur J Intern Med. 2016;36:36.

36. Zilberberg MD, Exuzides A, Spalding J, Foreman A, Jones AG, Colby C, Shorr AF. Hyponatremia and hospital outcomes among patients with pneumonia: a retrospective cohort study. BMC Pulm Med. 2008:8:16.

37. Bohl MA, Ahmad S, Jahnke H, Shepherd D, Knecht L, White WL, Little AS. Delayed hyponatremia is the most common cause of 30-day unplanned readmission after transsphenoidal surgery for pituitary tumors. Neurosurgery. 2016;78:84-90.

38. Zhou XY, Chen HL, Ni SS. Hyponatremia and short-term prognosis of patients with acute pulmonary embolism: a meta-analysis. Int J Cardiol. 2016;227:251-6.

39. Muratori R, Lisotti A, Fusaroli P, Caponi A, Gibiino G, Eusebi LH, Azzaroli F, Brighi N, Altimari G, Bazzoli F. Severe hypernatremia as a predictor of mortality after percutaneous endoscopic gastrostomy (PEG) placement. Dig Liver Dis. 2016:49:181-7.

40. Lindner G, Funk GC, Lassnigg A, Mouhieddine M, Ahmad SA, Schwarz C, Hiesmayr M. Intensive care-acquired hypernatremia after major cardiothoracic surgery is associated with increased mortality. Intensive Care Med. 2010;36:1718-23.

41. Sun T, Wu Q, Kan Q, Wan Y, Liu Z, Ma S, Zhang S, Zhang X, Wang H, Wang $Y$. The influence of hypernatremia on mortality in intensive care unit patients: a meta-analysis. Zhonghua Wei Zhong Bing Ji Jiu Yi Xue. 2014;26: 228-32.

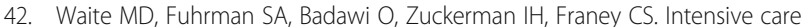
unit-acquired hypernatremia is an independent predictor of increased mortality and length of stay. J Crit Care. 2013;28:405-12.

43. Lindner G, Funk GC, Schwarz C, Kneidinger N, Kaider A, Schneeweiss B, Kramer L, Druml W. Hypernatremia in the critically ill is an independent risk factor for mortality. Am J Kidney Dis. 2007;50:952-7.

44. Alharfi IM, Stewart TC, Kelly SH, Morrison GC, Fraser DD. Hypernatremia is associated with increased risk of mortality in pediatric severe traumatic brain injury. J Neurotrauma. 2013;30:361-6.

45. Palevsky PM, Bhagrath R, Greenberg A. Hypernatremia in hospitalized patients. Ann Intern Med. 1996:124:197-203.

46. Snyder NA, Feigal DW, Arieff Al. Hypernatremia in elderly patients. A heterogeneous, morbid, and iatrogenic entity. Ann Intern Med. 1987;107: 309-19.

47. Long CA, Marin P, Bayer AJ, Shetty HG, Pathy MS. Hypernatraemia in an adult in-patient population. Postgrad Med J. 1991;67:643-5.

48. Alshayeb HM, Showkat A, Babar F, Mangold T, Wall BM. Severe hypernatremia correction rate and mortality in hospitalized patients. Am J Med Sci. 2011;341:356-60.

49. Arinzon Z, Feldman J, Peisakh A, Zuta A, Berner Y. Water and sodium disturbances predict prognosis of acute disease in long term cared frail elderly. Arch Gerontol Geriatr. 2005;40:317-26.

50. Molaschi M, Ponzetto M, Massaia M, Villa L, Scarafiotti C, Ferrario E. Hypernatremic dehydration in the elderly on admission to hospital. J Nutr Health Aging. 1997;1:156-60. 
51. Arampatzis S, Frauchiger B, Fiedler GM, Leichtle AB, Buhl D, Schwarz C, Funk GC, Zimmermann H, Exadaktylos AK, Lindner G. Characteristics, symptoms, and outcome of severe dysnatremias present on hospital admission. Am J Med. 2012;125:1125 e1-7.

52. Bataille S, Baralla C, Torro D, Buffat C, Berland Y, Alazia M, Loundou A, Michelet $\mathrm{P}$, Vacher-Coponat $\mathrm{H}$. Undercorrection of hypernatremia is frequent and associated with mortality. BMC Nephrol. 2014;15:37.

53. Maggs FG. The management of patients presenting with hypernatraemia: is aggressive management appropriate? Clin Med (Lond). 2014;14:260-3.

54. Ates I, Ozkayar N, Toprak G, Yilmaz N, Dede F. Factors associated with mortality in patients presenting to the emergency department with severe hypernatremia. Intern Emerg Med. 2016;11:451-9.

55. Falck-Ytter Y, Francis CW, Johanson NA, Curley C, Dahl OE, Schulman S, Ortel TL, Pauker SG, Colwell CW Jr, American College of Chest P. Prevention of VTE in orthopedic surgery patients: antithrombotic therapy and prevention of thrombosis, 9th ed: American College of Chest Physicians Evidence-Based Clinical Practice Guidelines. Chest. 2012;141:e278S-325S.

56. Johannesdottir SA, Horvath-Puho E, Dekkers OM, Cannegieter SC, Jorgensen JO, Ehrenstein V, Vandenbroucke JP, Pedersen L, Sorensen HT. Use of glucocorticoids and risk of venous thromboembolism: a nationwide population-based case-control study. JAMA Intern Med. 2013;173:743-52.

57. Higgins PD, Skup M, Mulani PM, Lin J, Chao J. Increased risk of venous thromboembolic events with corticosteroid vs biologic therapy for inflammatory bowel disease. Clin Gastroenterol Hepatol. 2015;13:316-21.

58. Kantar RS, Haddad AG, Tamim H, Jamali F, Taher AT. Venous thromboembolism and preoperative steroid use: analysis of the NSQIP database to evaluate risk in surgical patients. Eur J Intern Med. 2015;26:528-33.

59. Onusko E. Diagnosing secondary hypertension. Am Fam Physician. 2003; 67:67-74.

60. Mattsson C, Young WF Jr. Primary aldosteronism: diagnostic and treatment strategies. Nat Clin Pract Nephrol. 2006;2:198-208. quiz, 1 p following 30

61. Chae YK, Khemasuwan D, Dimou A, Neagu S, Chebrolu L, Gupta S, Carpio A, Kim J, Yun JH, Smyrlis A, et al. Inhibition of renin angiotensin axis may be associated with reduced risk of developing venous thromboembolism in patients with atherosclerotic disease. PLoS One. 2014;9:e87813.

62. Heit JA, Silverstein MD, Mohr DN, Petterson TM, O'Fallon WM, Melton LJ 3rd. Risk factors for deep vein thrombosis and pulmonary embolism: a population-based case-control study. Arch Intern Med. 2000;160:809-15.

63. Salahudeen AK, Doshi SM, Shah P. The frequency, cost, and clinical outcomes of hypernatremia in patients hospitalized to a comprehensive cancer center. Support Care Cancer. 2013;21:1871-8.

64. Mandala M, Falanga A, Roila F. Management of venous thromboembolism (VTE) in cancer patients: ESMO clinical practice guidelines. Ann Oncol. 2011; 22(Suppl 6):vi85-92.

65. Streiff MB, Bockenstedt PL, Cataland SR, Chesney C, Eby C, Fanikos J, Fogarty PF, Gao S, Garcia-Aguilar J, Goldhaber SZ, et al. Venous thromboembolic disease. J Natl Compr Cancer Netw. 2011;9:714-77.

66. Lyman GH, Khorana AA, Kuderer NM, Lee AY, Arcelus II, Balaban EP, Clarke $J M$, Flowers CR, Francis CW, Gates LE, et al. Venous thromboembolism prophylaxis and treatment in patients with cancer: American Society of Clinical Oncology clinical practice guideline update. J Clin Oncol. 2013;31: 2189-204.

67. Corona G, Giuliani C, Verbalis JG, Forti G, Maggi M, Peri A. Hyponatremia improvement is associated with a reduced risk of mortality: evidence from a meta-analysis. PLoS One. 2015;10:e0124105.

\section{Submit your next manuscript to BioMed Central and we will help you at every step:}

- We accept pre-submission inquiries

- Our selector tool helps you to find the most relevant journal

- We provide round the clock customer support

- Convenient online submission

- Thorough peer review

- Inclusion in PubMed and all major indexing services

- Maximum visibility for your research

Submit your manuscript at www.biomedcentral.com/submit
() BioMed Central 